\title{
Spin-dependent conductance statistics in systems with spin-orbit coupling
}

\author{
S L A de Queiroz \\ Instituto de Física, Universidade Federal do Rio de Janeiro, Caixa Postal 68528, 21941-972 Rio de Janeiro RJ, Brazil \\ Received: date / Revised version: date
}

\begin{abstract}
Spin-dependent partial conductances are evaluated in a tight-binding description of electron transport in the presence of spin-orbit (SO) couplings, using transfer-matrix methods. As the magnitude of SO interactions increases, the separation of spin-switching channels from non-spin-switching ones is gradually erased. Spin-polarised incident beams are produced by including a Zeeman-like term in the Hamiltonian. The exiting polarisation is shown to exhibit a maximum as a function of the intensity of SO couplings. For moderate site disorder, and both weak and strong SO interactions, no evidence is found for a decay of exiting polarisation against increasing system length. With very low site disorder and weak SO couplings a spin-filter effect takes place, as polarisation increases with increasing system length.
\end{abstract}

PACS. $05.60 . \mathrm{Gg}$ quantum transport - 72.25.-b spin polarized transport

\section{Introduction}

In this paper we study the statistics of the direct-current (DC) conductance of two-dimensional (2D) systems with spin-orbit (SO) interactions 1,2,3, in particular its spindependent properties and their dependence on various externally-imposed parameters.

Investigation of the interplay between the electron's spin magnetic moment and assorted properties of electronic systems is the subject of the emerging field of spintronics [4,5, 6]. Here we start from a tight-binding, spindependent Hamiltonian. Although this is a one-electron description, it is of course a full quantum-mechanical one. Thus the exclusion effects associated with Pauli's principle are implicit in the anticommutation rules obeyed by the respective single-particle creation and annihilation operators involved. In this context, our main aim is to give a quantitative account of the joint effects of exclusion, on the one hand, and spin flipping (enabled by SO couplings), on the other, upon electronic transport in disordered systems. Qualitatively, one will be asking whether an electron driven across a sample by a weak electric field will preferentially maintain the spin with which it was injected, or revert it via SO interactions; also of interest is the total current flowing through the sample in such circumstances.

We compare selected results of our calculations, especially those pertaining to spin polarisation, to those obtained for a variant of the similar (though classical) problem of directed flow with exclusion which mimicks the Pauli principle by allowing for conditional site doubleoccupation [7.

Section 2 below recalls some technical aspects of the transfer-matrix (TM) method used in our calculations. In
Sec. 3 we give numerical results for the statistics of spindependent conductances, both for spin-unpolarised systems and for the polarised case. For the latter we also investigate the behaviour of the polarisation itself, and of spin-correlation functions. In Sec. 4 we summarize and discuss our results.

\section{Theory}

The model one-electron Hamiltonian for this problem is

$$
\mathcal{H}=\sum_{i, \sigma} \varepsilon_{i} c_{i \sigma}^{\dagger} c_{i \sigma}+\sum_{\langle i, j\rangle} \sum_{\sigma, \sigma^{\prime}} V_{i j \sigma \sigma^{\prime}} c_{i \sigma}^{\dagger} c_{j \sigma^{\prime}}+\text { h.c. }
$$

where $c_{i \sigma}^{\dagger}, c_{i \sigma}$ are creation and annihilation operators for a particle with spin eigenvalues $\sigma= \pm 1$ at site $i$, and the self-energies $\varepsilon_{i}$ are independently-distributed random variables; $V_{i j \sigma \sigma^{\prime}}$ denotes the $2 \times 2$ spin-dependent hopping matrix between pairs of nearest-neighbour sites $\langle i, j\rangle$, whose elements must be consistent with the symplectic symmetry of SO interactions 8, 9. In Eq. (1) we describe SO couplings via an effective Hamiltonian with a single $(s$-like) orbital per site [3. Several possible forms may be considered for the hopping term, depending on whether one is specifically considering Rashba- or Dresselhaus- like couplings 3,10 , or (as is the case here) the focus is simply on the basic properties of systems in the symplectic universality class [11,12,13. 
Here we use the implementation of [11,12] for the hopping term, namely:

$$
V_{i j}=I+\mu i \sum_{k=x, y, z} V_{i j}^{k} \sigma^{k}=\left(\begin{array}{cc}
1+i \mu V_{i j}^{z} & \mu V_{i j}^{y}+i \mu V_{i j}^{x} \\
-\mu V_{i j}^{y}+i \mu V_{i j}^{x} & 1-i \mu V_{i j}^{z}
\end{array}\right) \text {, }
$$

where $I$ is the $2 \times 2$ identity matrix, $\sigma^{k}$ are the Pauli matrices, and $\mu$ gives the intensity of the SO coupling; below we consider the (real) $\left\{V_{i j}^{k}\right\}$ uniformly distributed in $[-1 / 2,1 / 2]$. Thus all energies are written in units of the $\mu \equiv 0$ nearest-neighbour hopping.

The $\varepsilon_{i}$ are taken from a random uniform distribution in $[-W / 2, W / 2]$.

The form Eq. (2) for the hopping term does not exhibit the explicit multiplicative coupling between momentum and spin degrees of freedom, characteristic of Rashba-like Hamiltonians [3, 10. In two dimensions one should not expect significant discrepancies between results from either type of approach, as long as one is treating systems without lateral confinement.

Since we shall not attempt detailed numerical comparisons to experimental data, the simplified formulation described above seems adequate for our purposes.

We apply the TM approach specific to tight-binding Hamiltonians like Eq. (11) [14, 15], with suitable adaptations for incorporating SO effects [16], considering a strip of the square lattice cut along one of the coordinate directions with periodic boundary conditions across. With SO couplings along the bonds, the site-dependent wavefunction amplitudes $a_{i k}$ ( $i, k$ being site coordinates) are now spinors, written on the basis of the eigenvectors of $\sigma^{z}$ as:

$$
a_{i k}=\left(\begin{array}{c}
a_{i k}^{\uparrow} \\
a_{i k}^{\downarrow}
\end{array}\right) \text {. }
$$

Our calculations of the two-terminal DC conductance follow the procedure described in [17, in which the TM is iterated and projected in such a way that the transmissivity matrix $t$ can be retrieved [17, 18, 19. From that the zero-field conductance $g$ is evaluated by the Landauer formula [19, 20

$$
g=\operatorname{Tr} t t^{\dagger}=\sum_{i, j}\left|t_{i j}\right|^{2},
$$

where the sum runs over all entry $(j)$ and exit $(i)$ channels.

In this scheme one considers a disordered system described by Eq. (11), with $N$ sites across and length $M$ sites, connected to pure leads at both ends (that is, where all $\left.\varepsilon_{i} \equiv 0, \mu \equiv 0\right)$. Eq. (44) gives the total conductance, i.e., considering both spin directions. The methods of [17] were used to evaluate the probability distribution of the total conductance [21,22,23,24], at the metal-insulator transition for $2 \mathrm{D}$ systems with $\mathrm{SO}$ couplings.

For spin-dependent properties one considers the partial conductances $g_{\sigma \sigma^{\prime}}$, where

$$
g_{\sigma \sigma^{\prime}}=\sum\left|t_{\sigma \sigma^{\prime}}\right|^{2}
$$

with $t_{\sigma \sigma^{\prime}}$ being the transmission coefficient from the left lead with spin $\sigma^{\prime}$ to the right lead with spin $\sigma$ [25,26]. The sum runs over all such pairs of channels with fixed $\sigma, \sigma^{\prime}$.

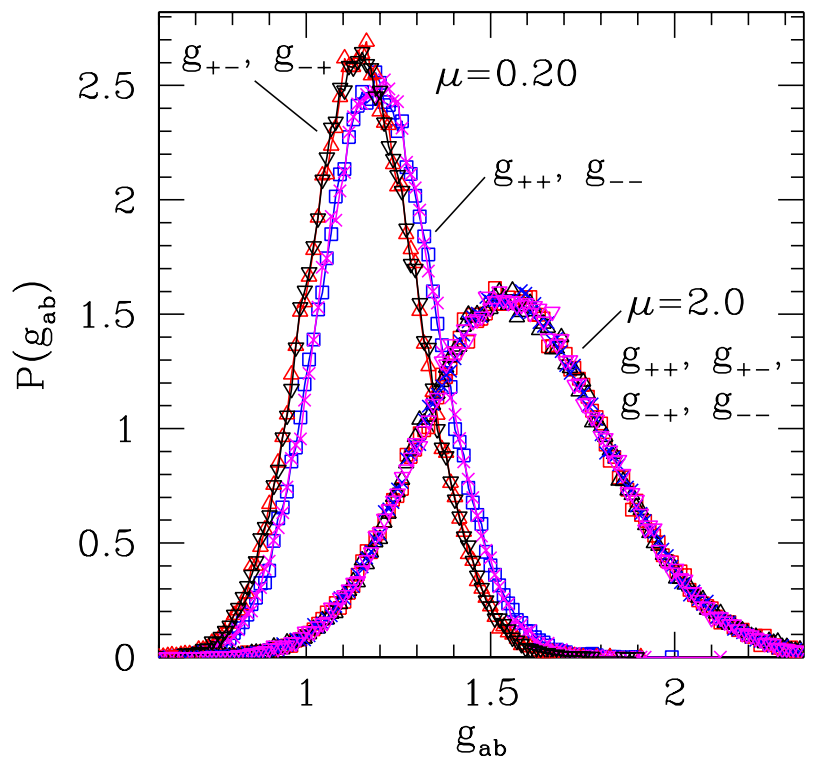

Fig. 1. Probability distributions for partial conductances $g_{a b}$, $a, b= \pm$ of system with $M=N=40$ sites for energy $E=0$, $W=3.0$, with $\mu=0.20$ (leftmost curves) and 2.0, see Eqs. (1) and (2), both in the metallic phase. The incident beams are spin-unpolarised. $N_{s}=10^{5}$ independent samples for each case.

\section{Numerics}

\subsection{Introduction}

Here we take $E=0$, corresponding to the Fermi energy of a non-disordered system with $W=\mu \equiv 0$. At $E=0, \mu=$ 2.0 the critical disorder for the metal-insulator transition on a square lattice is estimated as $W_{c}=8.55$ (5) [12.

For each set of $E, \mu$, and $W$ studied we generally take $N_{s}=10^{5}$ independent realizations of disorder, which enables us to obtain smooth curves for the distributions of conductances and associated quantities.

\subsection{Unpolarised beams}

The plane wave-like incident states used are straightforward adaptations of those used for the spinless case [17, with the spinors of Eq. (3) having equal components. So in this case the incident beam is fully spin-unpolarised.

We now specialise to the conducting phase. With $E=$ $0, \mu=2.0$ we took $W=3$, well within the metallic phase. Then, keeping $W$ fixed we lowered the SO coupling by an order of magnitude, making $\mu=0.2$. For this $\mu$ the critical disorder at the centre of the unperturbed band is $W_{c} \approx 5.3$. The results for the distributions of the $g_{a b}$ are displayed in Fig. 1, confirming to a very good extent their expected Gaussian shape [22,27].

While for $\mu=2.0$ all four curves coincide (we checked that this also happens at the metal-insulator transition for this $\mu$, namely $W_{c}=8.55$ ), there is a split between the spin-conserving and spin-flipping groups at $\mu=0.20$ 
where the SO effects are weaker. For the lower $\mu$ the peaks of the $(+-),(-+)$ group are located at a value of $g$ some $3.7 \%$ less than those of $(++),(--)$. For comparison, for $\mu=2.0$ the (non-systematic) spread among the peak locations for all four distributions is of order $0.3 \%$.

\subsection{Spin-polarised beams}

When attempting to represent spin-polarised mixtures of spins with the methods of [17, the following procedures may be devised in order to set a specific incoming polarisation: either (i) impose unequal amplitudes for $\uparrow$ and $\downarrow$ spinor components on the input state vectors, see Eq. (3), or (ii) suitably modify the Hamiltonian Eq. (1) while keeping the input vectors unconstrained.

We have considered procedure (i); this corresponds to a straightforward generalization of well-known procedures used in variants of the classical problem of directed flow with exclusion, which include conditional allowance of site double-occupation (mimicking the Pauli principle) 7 . Overall, we found that the effect of using such constrained input vectors amounts to a modulation of the channelspecific conductances by the respective incoming and outgoing polarisations, the latter being predicted to equal the former on the basis of the time-reversal symmetry of the Hamiltonian 19. Though numerical data have been found to be compatible with such conservation, it is not clear at this point how one might extract additional physical insight from the resulting picture.

We thus concentrated on procedure (ii). One can add spin-dependent energies to Hamiltonian Eq. (1), e.g., by introducing an external magnetic field as done in [25] in a similar context. In this case, at energy $E$ there are more open propagation channels $\left(\varepsilon_{i}(\sigma)>E\right)$ for one spin species than for the other. Analysis of level-spacing statistics has shown that, upon application of such field, the universality class of the problem changes from the Gaussian symplectic ensemble to that of the Gaussian unitary ensemble [25]. This is to be expected from symmetry considerations 28]. Furthermore, conservation of average polarisation is now not guaranteed since time-reversal symmetry has been broken by the magnetic field [19].

At least two possible implementations can be suggested: (i) a field acting only on the left lead [25], or (ii) a spindependent chemical potential acting on every site of the sample. For simplicity here we stick to option (i).

With $\varepsilon_{i}^{0}$ being the self-energy of an orbital on the left lead in the absence of field, we establish our Zeemanlike energy origin such that $\varepsilon_{i}(+)=\varepsilon_{i}^{0}, \varepsilon_{i}(-)=\varepsilon_{i}^{0}+\Delta$ $(\Delta>0)$, so propagation of plus spins is favored. Below we make $\Delta=3.75$. As the unperturbed single-electron band spans the interval $-4 \leq E \leq 4$ in the square-lattice geometry, this induces a significant spin imbalance. We also considered other values in the range $1.0 \leq \Delta \leq 4.0$, with qualitatively similar results.

Here we always take the incoming beams as fully spinunpolarised, so any resulting polarisation is driven by the Zeeman-like gap $\Delta$.

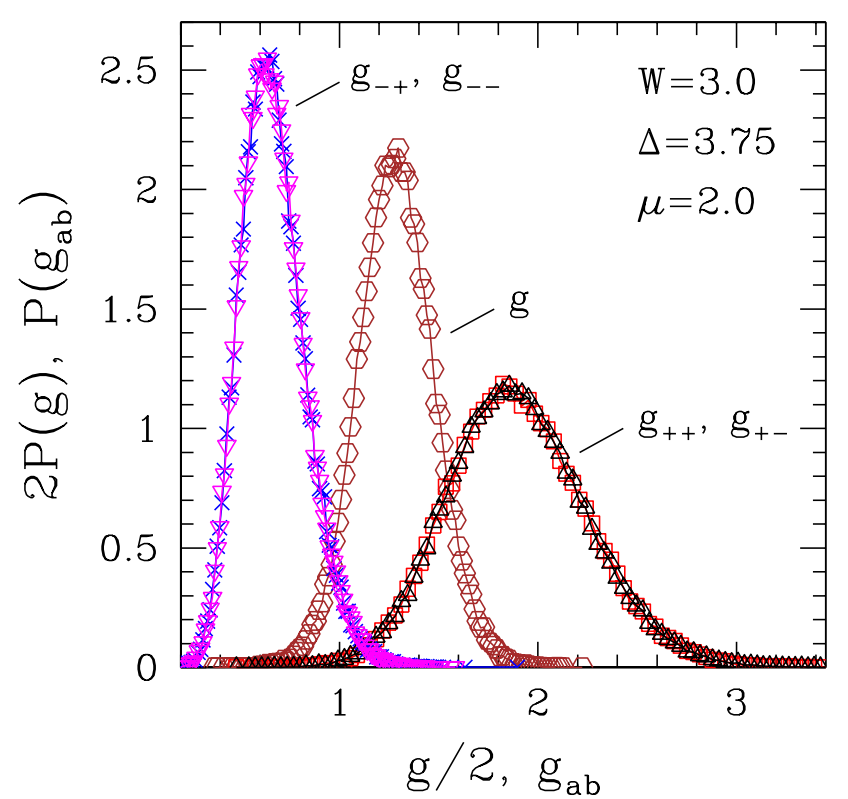

Fig. 2. Probability distributions for total $(g)$ and partial $\left(g_{a b}\right.$, with $a, b= \pm$ ) conductances of system with $M=N=20$ sites for energy $E=0$, in the conducting phase with $\mu=2.0$, $W=3.0$, and $\Delta=3.75 . N_{s}=10^{5}$ independent samples.

As in Section 3.2 we specialize to the conducting phase, so the conductance distributions are expected to be close to Gaussian [22,27]. In this context the central estimates, denoted by $\langle\cdots\rangle$, and error bars given for conductances and related quantities below correspond, respectively, to mean value and RMS deviation of the associated distributions.

Figs. 2 and 3 show the distributions for total and partial conductances, and should be compared, respectively, with the rightmost and leftmost groups of Fig. 1, which both correspond to $\Delta=0$. For $\mu=2.0$ (Fig. 2) channel asymmetry is manifest in that there is a grouping of distributions by exiting channels, respectively for majority $[(+,+)$ and $(+,-)]$ and minority $[(-,+)$ and $(-,-)]$ spins, while for $\Delta=0$ all four distributions coincide. Note that this is not the same pairing exhibited by the $\mu=0.2$ curves in Fig. 11 where the groupings are spinflipping $[(+,-)$ and $(-,+)]$ and spin-conserving $[(+,+)$ and $(-,-)]$, both due to up-down symmetry.

For $\mu=0.2$ (Fig. 3) all four channels are fully split, as up-down symmetry is now absent. However, a tendency towards the same grouping of Fig. 2 still holds.

The polarisation $\mathcal{P}$ of the exiting current can be evaluated $[26$ as

$$
\mathcal{P}=\frac{g_{++}+g_{+-}-\left(g_{--}+g_{-+}\right)}{g_{++}+g_{+-}+g_{--}+g_{-+}},
$$

see Eq. (5).

In Fig. 4 the probability distributions for $\mathcal{P}$ are shown, for systems with fixed width and varying aspect ratios $M / N$. The distributions broaden out as the system becomes more wire-like, and develop a more pronounced 


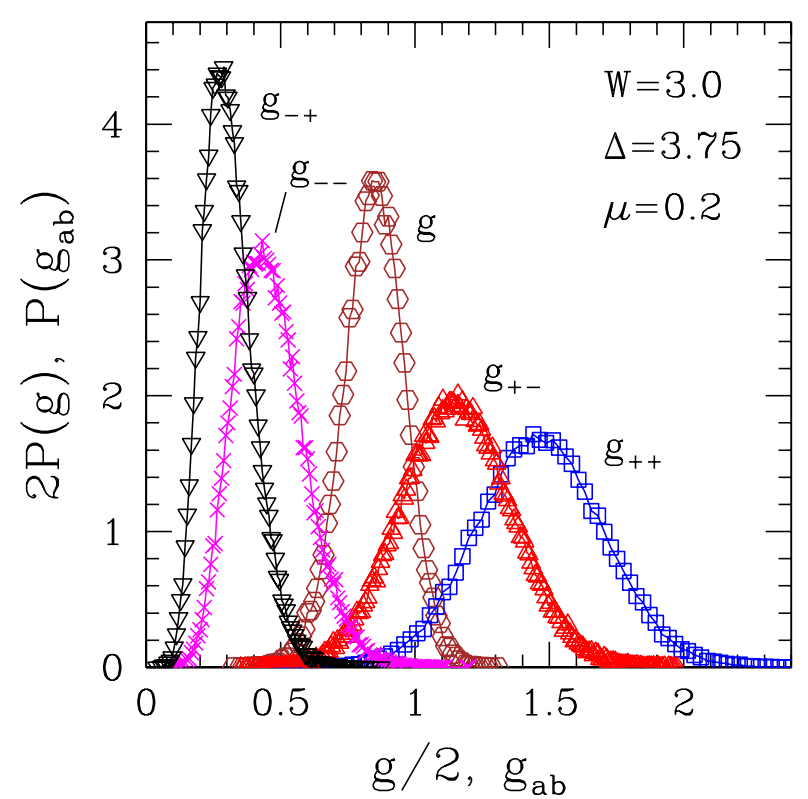

Fig. 3. Probability distributions for total $(g)$ and partial $\left(g_{a b}\right.$, with $a, b= \pm)$ conductances of system with $M=N=20$ sites for energy $E=0$, in the conducting phase with $\mu=0.2$, $W=3.0$, and $\Delta=3.75 . N_{s}=10^{5}$ independent samples.

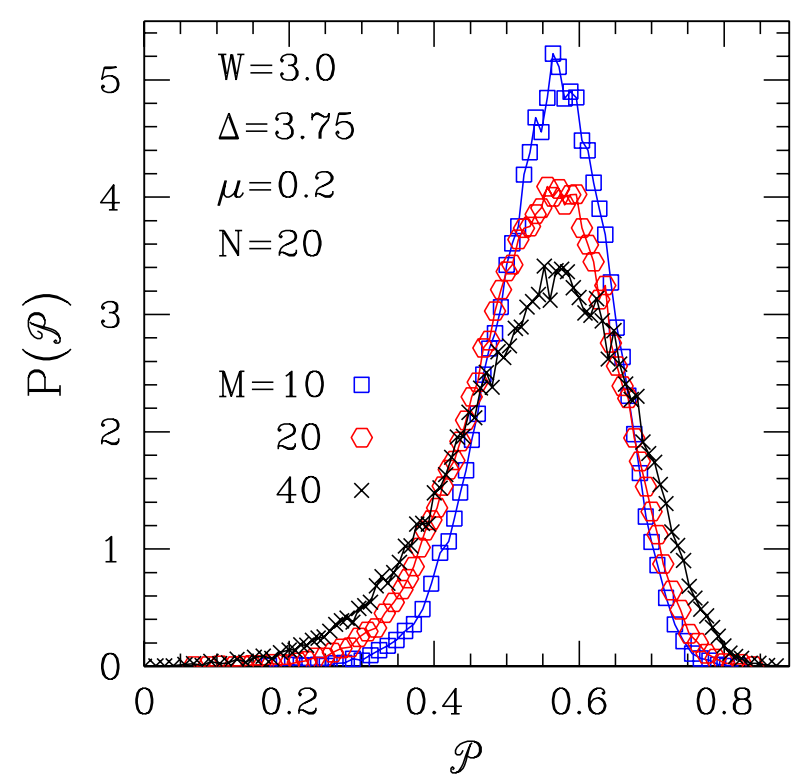

Fig. 4. Probability distributions for exiting polarisation $\mathcal{P}$ at right end of systems of fixed width $N=20$ sites, and varying length $M=10,20$, and 40 sites. All for $E=0$, in the conducting phase with $\mu=0.2, W=3.0$, and $\Delta=3.75 . N_{s}=10^{5}$ independent samples. negative skew. On the other hand the position of their peak value remains essentially unchanged. So, despite the Hamiltonian not being invariant under time reversal, we see indications that polarisation of the beam is conserved in this case.

While Fig. 4 is for $\mu=0.2$, we have found qualitatively very similar outcomes for larger $\mu$, in particular the stability of the peak location for $P(\mathcal{P})$ against varying $M$. Having this in mind, we kept $M=10$ fixed and varied several other parameters. In this way we are reasonably sure to retain the main qualitative features of the quantities under study, while the spread of distributions is kept lower than for larger $M$.

Fig. 5 shows, for fixed $\Delta=3.75$, the variation of the average exiting polarisation $\langle\mathcal{P}\rangle$, and total conductance $\langle g\rangle$, against the intensity $\mu$ of SO coupling. The maximum exhibited in panel (a) for $\mu \approx 0.75$ will be discussed in detail below. Note however that the difference between minimum and maximum values of $\langle\mathcal{P}\rangle$ is only some $30 \%$ of the largest, on account of the largish value of $\Delta$. On the other hand, panel (b) shows that increasing SO effects lead to monotonically increasing total conductance, for fixed $\Delta$.

The leftmost points of panel (a), (b) of Fig. 5 correspond to $\mu=0.01$ which most probably is in the insulating phase; approximate calculations indicate that for $W=3.0$ the metal-insulator boundary lies at $\mu \approx 0.06$. Even so, such point in $(W, \mu)$ space would have an associated localization length much larger than the current system's size. Thus we believe it is justifiable to present data for this point together with those unequivocally belonging in the conducting phase.

The unusual behaviour exhibited by $\langle\mathcal{P}\rangle$ against $\mu$ warrants further investigation. In order to check on the possible influence of finite-size effects we ran simulations for $N=40$ and 60 , keeping $M=N / 2$ to preserve the aspect ratio, and spanning the same set of $\mu$ values as in Fig. 5 . Except for very weak SO coupling, $\mu=0.01$, where the centre of the distribution of $\mathcal{P}$ shifts to smaller values by a significant amount upon increasing $N$, we see no shift for the corresponding centres at larger $\mu$ as $N$ increases (in which case the only noticeable effect is that the peaks become sharper). In conclusion, the non-monotonic variation of $\langle\mathcal{P}\rangle$ against $\mu$ observed in Fig. 5 does not appear to be an artifact from finite sample dimensions.

To see the physical origin of the polarisation maximum one must first follow the evolution, for fixed exit channel, of the split between spin-conserving and spin-flipping partial conductances; then one must consider how the difference between said splits evolves. We define

$$
\delta g_{+} \equiv g_{++}-g_{+-} ; \quad \delta g_{-} \equiv g_{--}-g_{-+},
$$

where the $\mu$-dependence of all quantities is implicit.

Firstly, both $\delta g_{+}$and $\delta g_{-}$are positive at small $\mu$ (see Fig. 3), and vanish upon increasing $\mu$ (see Fig. 2). Secondly, for $\Delta>0$ one has $\delta g_{+}>\delta g_{-}$(see Fig. 3 for an example), because: (i) the external field generally favours upward-pointing spins, so $g_{++}>g_{--}$, while (ii) for any $\mu$ and for fixed exit channel, the ratio of spin-flipping to 


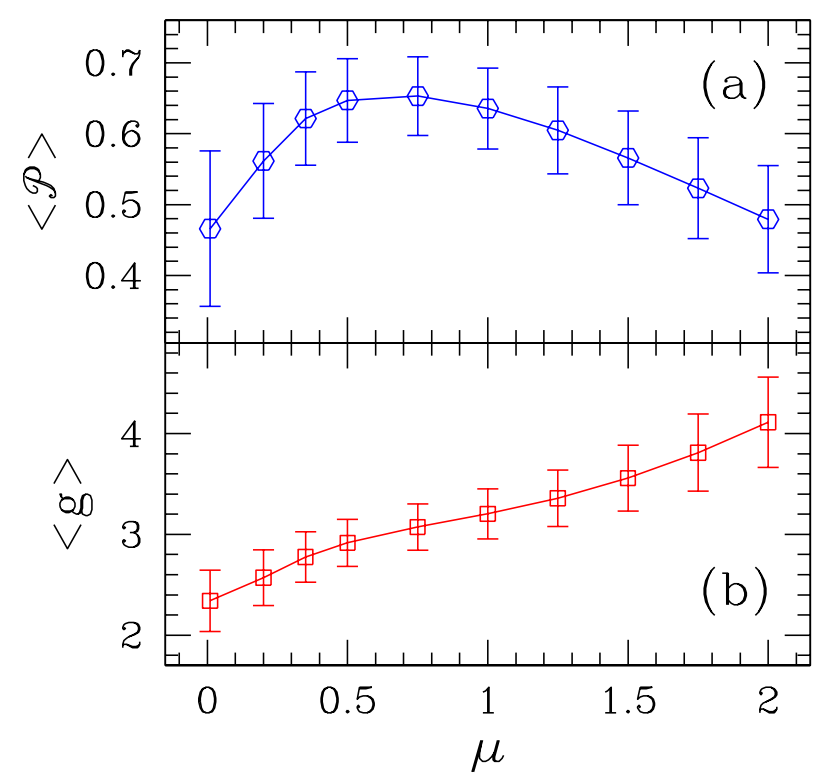

Fig. 5. For $E=0$ with $W=3.0, \Delta=3.75$, and varying $\mu$, panel (a) shows average exiting polarisation $\langle\mathcal{P}\rangle$ at right end, and (b) gives average total conductance $\langle g\rangle$, of systems of fixed width $N=20$, length $M=10$ sites. All for $N_{s}=10^{5}$ independent samples.

spin-conserving conductance is nearly independent of exit channel, as will be shown in panel (b) of Fig. 6] below. Eq. (6) can be rewritten as

$$
\mathcal{P}=\frac{2\left(g_{++}-g_{--}\right)}{\Sigma}+\frac{\left(\delta g_{-}-\delta g_{+}\right)}{\Sigma}
$$

with $\Sigma \equiv g_{++}+g_{+-}+g_{--}+g_{-+}$.

Panel (a) of Fig. [6 shows how each of the two terms on the right-hand side of Eq. (8) behaves individually against $\mu$. The contribution given by the first (spin-conserving) one follows the expected decreasing trend upon increasing $\mu$, in accord with the idea that SO effects tend to equalise up- and downward-pointing spins. On the other hand, both $\delta g_{+}$and $\delta g_{-}$vanish for $\mu \approx 0.75$ (see also panel (b) of Fig. 6). Clearly the initially-growing behaviour of $\mathcal{P}$ is due to the negative, and diminishing, contribution of the second term.

We now turn to cases in which the effects of site disorder are suppressed. Still with $E=0$ and $\Delta=3.75$ we took $W=10^{-5}$, which is essentially zero for practical purposes here. We considered $P(\mathcal{P})$ for systems of width $N=20$ sites across, and lengths $M=10,20$, and 40. The picture for $\mu=2.0$ was qualitatively and quantitavely very similar to that given in Fig. 4 for $W=3.0, \mu=0.2$ : peak positions and RMS widths respectively $0.54-0.55$ and $0.04-0.06$ $\left(W=10^{-5}, \mu=2.0\right), 0.54-0.56$ and $0.08-0.12(W=3.0$, $\mu=0.2)$

On the other hand, for weak SO couplings $\mu=0.2$, as shown in Fig. 7, it is seen that for this combination of parameters the system can function as a spin filter [26], with the beam polarisation increasing as it propagates. Quan-

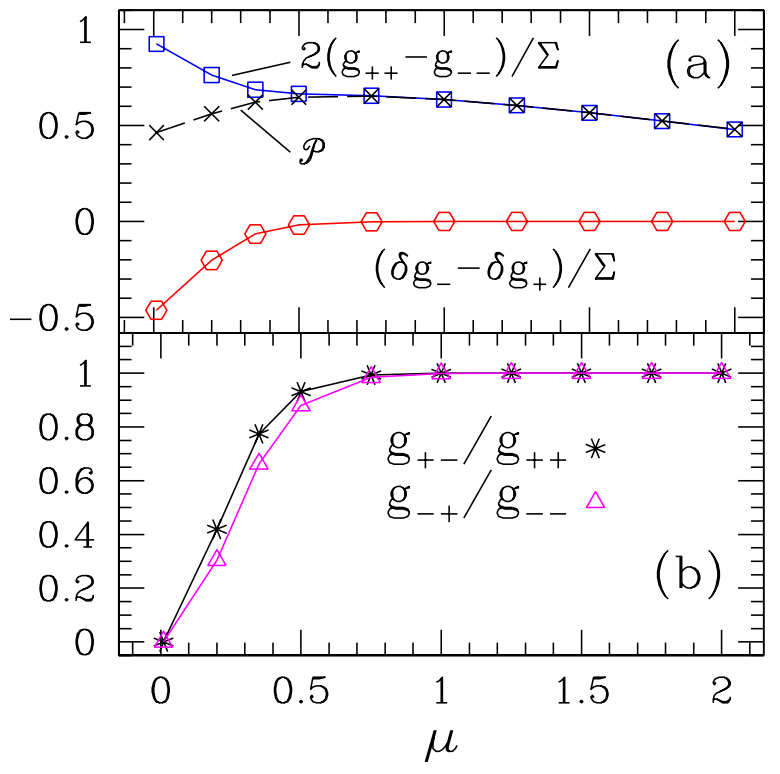

Fig. 6. Panel (a): plots of the individual contributions from each of the terms on the right-hand side of Eq. (8). The resulting polarisation $\mathcal{P}$ is shown (crosses joined by dashed line). Panel (b): ratios of spin-flipping to spin-conserving conductances according to exit channel. Error bars are omitted for clarity. All for $E=0$ with $W=3.0, \Delta=3.75, N=20$, $M=10, N_{s}=10^{5}$ independent samples.

titatively, $\langle\mathcal{P}\rangle(M=40)=0.774(14),\langle\mathcal{P}\rangle(M=10)=$ $0.741(8)$.

Taking strong SO coupling $\mu=2.0$, the spin-filter effect becomes essentially undiscernible: one gets $\langle\mathcal{P}\rangle(M=$ $40)=0.541(75),\langle\mathcal{P}\rangle(M=20)=0.540(65),\langle\mathcal{P}\rangle(M=$ 10) $=0.541(55)$.

In this region of parameter space one can capture the decay of the spin-spin correlation function [10]

$$
F_{z z}(L)=\left\langle\sigma^{z}(0) \sigma^{z}(L)\right\rangle,
$$

which is the conditional probability to find an electron with spin $\sigma^{z}$ at $x=L$, for an incident electron at $x=0$ with $\sigma^{z}= \pm 1$. In terms of the $g_{\sigma \sigma^{\prime}}$ of Eq. (5), this is:

$$
F_{z z}=\frac{1}{2}\left\langle\frac{g_{++}-g_{-+}}{g_{++}+g_{-+}}+\frac{g_{--}-g_{+-}}{g_{--}+g_{+-}}\right\rangle \text {. }
$$

The spin relaxation length $\Lambda_{s}[10$ is defined by assuming an exponential decay for the correlation function, $F_{z z}(L) \propto \exp \left(-L / \Lambda_{s}\right)$. Fig. 8 displays data for $E=0$, $\Delta=3.75, W=10^{-5}, \mu=0.2$, from which a fit to the above expression gives $\Lambda_{s}=22(2)$. Of course this is intended mostly for illustration, since here one has only three data points. Nevertheless, a semi-quantitative analysis of such results can prove enlightening. For example, keeping the same set of parameters and system sizes but making $W=3.0$ gives $\Lambda_{s}=9.0(2)$; if one then keeps $W=3.0$ and makes $1.0 \leq \Delta \leq 4.0, \Lambda_{s}$ remains within $10 \%$ of that. However, for strong SO coupling $\mu=2.0$, spin relaxation takes place at very short distances even for 


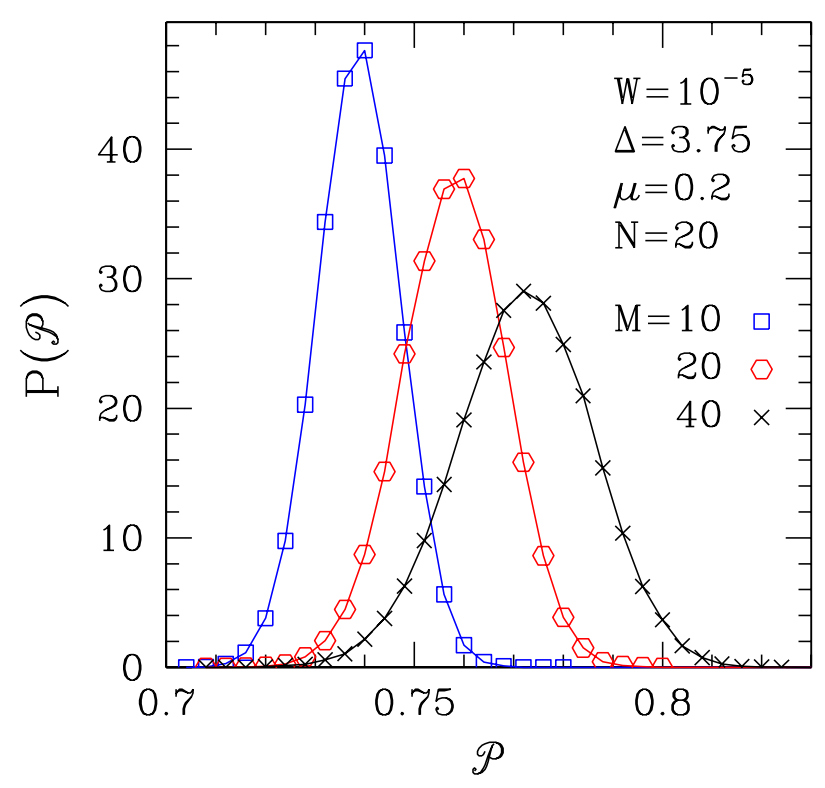

Fig. 7. Probability distributions for exiting polarisation $\mathcal{P}$ at right end of systems of fixed width $N=20$ sites, and varying length $M=10,20$, and 40 sites. All for $E=0$, in the conducting phase with $\mu=0.2, W=10^{-5}$, and $\Delta=3.75 . N_{s}=10^{5}$ independent samples.

very low site disorder. In this case, already for $M=10$ the distributions of $F_{z z}(M)$ remain essentially centred around zero for all sets of physically plausible parameters used here.

\section{Discussion and Conclusions}

The maximum exhibited by $\langle\mathcal{P}\rangle$ against varying $\mu$, shown in panel (a) of Fig. 5, can be explained with the help of Eqs. (7) and (8), and Fig. 6. The explanation given in Section 3.3 works because:

(i) $\delta g_{+}, \delta g_{-}$vanish continuously with increasing $\mu$, for $\Delta \geq 0$

(ii) $\delta^{2} g \equiv \delta g_{+}-\delta g_{-}>0$ for $\Delta>0$ and suitably small $\mu$.

We do not know of a rigorous proof for either of statements (i) or (ii). A plausibility argument for (i) is that since both $\delta g_{+}$and $\delta g_{-}$are positive for $\mu=0$, and given the spin-switching character of $\mu$, the alternative for their vanishing with growing $\mu$ would be their turning negative. This would be at odds with the notion of SO interactions as inducing channel equalisation. As for (ii), we recall that (1) for $\Delta>0$ one should have $g_{++}>g_{--}$; (2) the data in panel (b) of fig. 6] show that the fractional variations $\delta g_{+} / g_{++}$and $\delta g_{-} / g_{--}$are roughly equal at any given $\mu$, which appears reasonable. Points (1) and (2) help one understand (ii).

In summary, the maximum of $\langle\mathcal{P}\rangle$ against intensity of SO interactions is structurally linked to the behaviour of spin-flipping and spin-conserving conductances, and some of their combinations, namely $\delta g_{+}, \delta g_{-}$, and the secondorder difference $\delta^{2} g$. As this is a phenomenon taking place

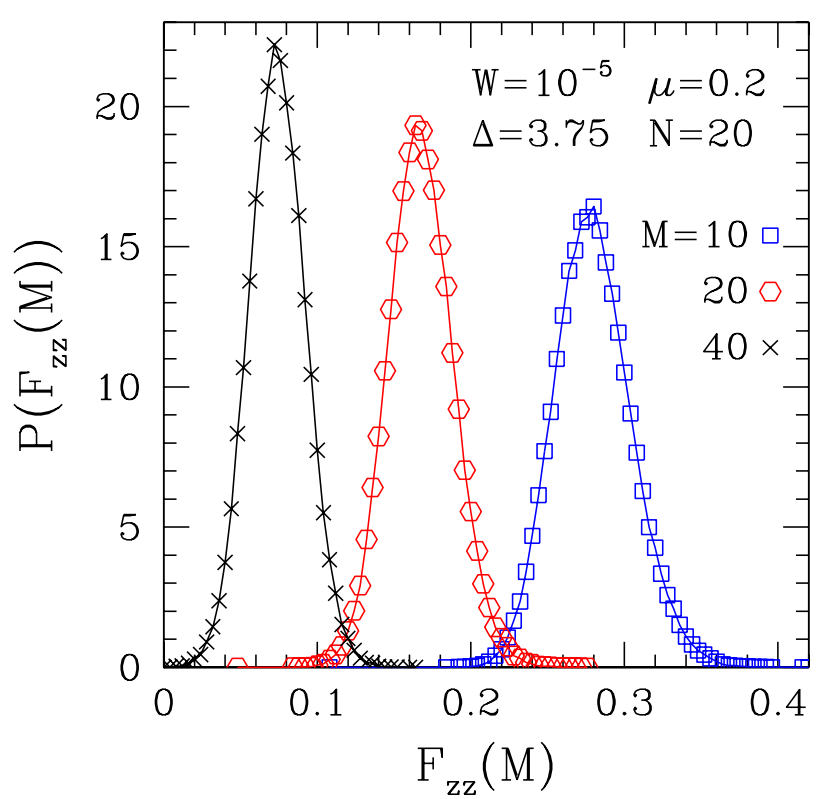

Fig. 8. Probability distributions for correlation function $F_{z z}(M)$ [see Eqs. (9), (10)] between left and right end of systems of fixed width $N=20$ sites, and varying length $M=10$, 20, and 40 sites. All for $E=0$, in the conducting phase with $\mu=0.2, W=10^{-5}$, and $\Delta=3.75 . N_{s}=10^{5}$ independent samples.

in spin space, such feature is expected to be always present, irrespective of the detailed form taken by the SO couplings, system size, and field strength.

The spin-dependent parameter $\Delta$ added to the Hamiltonian in Sec. 3.3 breaks time-reversal symmetry. So there is no guarantee that results pertaining to that Section should exhibit conservation of spin polarisation [19. Nevertheless, we have found for moderately strong site disorder $W$, and for both weak and strong SO couplings, that the average spin polarisation is kept constant along significant distances within the system (though of course, variations associated with a very large characteristic length cannot be ruled out). Moreover, in the special case of low site disorder and weak SO interactions, we have evinced spin-filter behaviour (see Fig. 7), with spin polarisation increasing with distance from the injection edge.

The classical model studied in 7, where SO effects are mimicked by so-called "spin-flipping" sites, always exhibits polarisation decay against distance. This would be in line with entropic interpretations of a spin-flipping agent contributing towards equalising spin populations in an initially spin-ordered beam. In contrast with that, here we have not found combinations of parameters which produce such behaviour. So one sees that the symmetries imbedded in the quantum-mechanical formulation used here are enough to counteract any such trend, even though the initial beams are prepared in as close a manner as possible in either case.

Investigation of the spin-spin correlation function [10, see Fig. 8, yields significant information on the characteristic decay length $\Lambda_{s}$, for low and moderate site dis- 
order, provided the SO coupling is weak. For strong SO interactions correlation decay takes place over short distances, comparable to the lattice spacing. It would then appear that the polarisation results for the classical process studied in [7, and their always-decaying behaviour against distance, find a closer qualitative correspondence here with the properties of the correlation function, rather than those of its namesake.

The author thanks F Pinheiro, C Lewenkopf, T Ohtsuki, and $\mathrm{J} \mathrm{T}$ Chalker for helpful discussions, and the Brazilian agencies CNPq (Grant No. 303891/2013-0) and FAPERJ (Grant No. E-26/102.348/2013) for financial support.

\section{References}

1. S. Hikami, A. I. Larkin, Y. Nagaoka, Prog. Theor. Phys. 63, 707 (1980)

2. G. Bergmann, Phys. Rep. 107, 1 (1984)

3. T. Ando, Phys. Rev. B 40, 5325 (1989)

4. I. Zutić, J. Fabian, S. Das Sarma, Rev. Mod. Phys. 76, 323 (2004)

5. S.D. Bader, S.S.P. Parkin, Annual Reviews of Condensed Matter Physics 1, 71 (2010)

6. V. Baltz, A. Manchon, M. Tsoi, T. Moriyama, T. Ono, Y. Tserkovnyak, Rev. Mod. Phys. 90, 015005 (2018)

7. S. L. A. de Queiroz, R. B. Stinchcombe, Phys. Rev. E 95, $042121(2017)$

8. A. M. S. Macêdo, J. T. Chalker, Phys. Rev. B 46, 14985 (1992)

9. R. Merkt, M. Janssen, B. Huckestein, Phys. Rev. B 58, 4394 (1998)

10. T. Kaneko, M. Koshino, T. Ando, Phys. Rev. B 78, 245303 (2008)

11. S N. Evangelou, T. Ziman, J. Phys. C: Solid State Phys. 20, L235 (1987)

12. S. N. Evangelou, Phys. Rev. Lett. 75, 2550 (1995)

13. Y. Asada, K. Slevin, T. Ohtsuki, Phys. Rev. B 70, 035115 (2004)

14. J.-L. Pichard, G. Sarma, J. Phys. C: Solid State Phys. 14 L127 (1981)

15. J.-L. Pichard, G. Sarma, J. Phys. C: Solid State Phys. 14 L617 (1981)

16. A. Yamakage, K. Nomura, K.-I. Imura, Y. Kuramoto, Phys. Rev. B 87, 205141 (2013)

17. J. B. Pendry, A. MacKinnon, P. J. Roberts, Proc. Roy. Soc. Series A 437, 67 (1992)

18. A. P. Taylor, A. MacKinnon, J. Phys.: Condens. Matter 14, 8663 (2002)

19. Y. V. Nazarov, Y. M. Blanter, Quantum Transport (Cambridge University Press, 2009)

20. R. Landauer, Phil. Mag. 21, 863 (1970)

21. M. Rühlander, P. Markoš, C. M. Soukoulis, Phys. Rev. B $64193103(2001)$

22. P. Markoš, in Anderson Localization and Its Ramifications, Lecture Notes in Physics Vol. 630, edited by T. Brandes and S. Kettemann (Springer, 2003); cond-mat/0211037

23. T. Ohtsuki, K. Slevin, B. Kramer, Physica E 22, 248 (2004)

24. P. Markoš, L. Schweitzer, J. Phys. A: Math. General 39 3221 (2006)
25. J.-I. Ohe, M. Yamamoto, T. Ohtsuki, Phys. Rev. B 68 $165344(2003)$

26. J.-I. Ohe, M. Yamamoto, T. Ohtsuki, J. Nitta, Phys. Rev. B 72 041308(R) (2005)

27. J.-L. Pichard, in Quantum Coherence in Mesoscopic Systems, NATO ASI Series B, Vol 254, edited by B. Kramer (Plenum, 1991)

28. C. W. J. Beenakker, Rev. Mod. Phys. 69, 731 (1997) 